\title{
Thiamine Deficiency (Beriberi) Induced Polyneuropathy and Cardiomyopathy: Case Report and Review of the Literature
}

\author{
Charles Qiming Cui ${ }^{\mathrm{a}}$, Ling L. Qiu ${ }^{\mathrm{b}, \mathrm{c}}$
}

\begin{abstract}
Thiamine, also known as vitamin B1, is an essential nutrient required for all living tissues. It can be found in high concentration inside the heart, kidneys, brain and skeletal muscles. A severe depletion is not commonly seen, except in cases of inadequate nutrition and/or alcoholism. The major manifestation of thiamine deficiency involves the nervous (dry beriberi, or neuropathy and/or WernickeKorsakoff syndrome) and cardiovascular (wet beriberi or cardiomyopathy) systems. This report details the case of a female patient with severe thiamine deficiency secondary to dysphagia caused by radiation injury. She developed debilitating polyneuropathy and cardiomyopathy with decreased ejection fraction. Early recognition and adequate thiamine replacement led to a full recovery in this case. In addition, we will discuss the potential benefits of thiamine supplementation in treating congestive heart failure.
\end{abstract}

Keywords: Thiamine; Beriberi; Heart failure

\section{Introduction}

Thiamine deficiency, or beriberi, is commonly seen in developing countries due to inadequate nutrition and alcohol abuse. There have been a few cases reported in the United States in recent years. Because the presentation of thiamine deficiency involves multiple systems, it can be easily misdiagnosed. Early recognition and appropriate treatment are critical and can lead to a full recovery of the illness. Here

\footnotetext{
Manuscript accepted for publication April 11, 2014

${ }^{\mathrm{a}}$ University of Louisville School of Medicine, Louisville, Kentucky, USA

${ }^{\mathrm{b}}$ Norton Healthcare at Louisville Kentucky, Louisville, Kentucky, USA

${ }^{\mathrm{c} C}$ Corresponding author: Ling Qiu, University of Louisville School of Medicine, Louisville, Kentucky, USA.

Email: lingqiu2004@yahoo.com
}

doi: http://dx.doi.org/10.14740/jmc1780w we discuss the case of a 20-year-old female who developed beriberi syndrome. A full recovery was achieved after thiamine replacement.

Literature in this review was found via PubMed-assistant search. We will discuss basic thiamine metabolism, its biological function and the pathophysiology of beriberi. In addition, we will review recent literatures regarding the potential benefits of thiamine supplement for the treatment of congestive heart failure (CHF).

\section{Case Report}

A 20-year-old white female with a history of papillary thyroid carcinoma, status post total thyroidectomy and recent 12-week radiation therapy for retropharyngeal mass showed up in the ER with progressive bilateral lower extremity weakness and paresthesia, inability to walk, chest palpitations and shortness of breath. Her symptoms gradually developed over the past few weeks with increased intensity on the day of presentation. On further questioning, she revealed progressive difficulty in swallowing for months due to dry mouth and choking spells, and credited this with a subsequent decrease in oral intake and weight loss. She was recommended for gastrostomy tube support but refused medical intervention.

On physical exam, she was a young lady with ill appearance and low body weight. She was in moderate distress due to her shortness of breath. Her voice was so weak that she could not finish a sentence without taking a break. She had normal temperature with blood pressure at 130/105; her heart rate exhibited sinus rhythm of 130 beats per minute on the monitor. Respiratory rate was 30 breaths per minute with $94 \%$ saturation on room air. Abnormal physical findings included ptosis, nystagmus, and tachycardia with a soft $3 / 6$ systolic murmur heard best at the apex and fine rales at the base of her lung bilaterally. She also had low muscle strength on all four extremities symmetrically, especially on both legs. Her legs were $2 / 5$ with no movement against gravity and absent patellar and ankle reflexes on both sides. She does not use alcohol or tobacco and resides with her mother. Laboratory studies revealed hemoglobin of $15 \mathrm{~g} / \mathrm{dL}$ 
(12.0 - 16.0), hematocrit 43.1\% (38.0-47.0), white count at $3.9 \mathrm{THOU} / \mu \mathrm{L}(4.00-11.00)$ and platelets at $279 \mathrm{THOU} / \mu \mathrm{L}$ (150 - 375). Sodium was at $136 \mathrm{mEq} / \mathrm{L}(137-145)$, with potassium $3.1 \mathrm{mEq} / \mathrm{L}(3.5$ - 5.1), chloride $97 \mathrm{mEq} / \mathrm{L}$ (98 - 109), carbon dioxide $26 \mathrm{mEq} / \mathrm{L}(22.0$ - 30.0), BUN $10 \mathrm{mg} / \mathrm{dL}$ (7 - 25), creatinine $0.53 \mathrm{mg} / \mathrm{dL}(0.70$ - 1.30), glucose $111 \mathrm{mg} /$ dL (70 - 100), calcium 8.9 mg/dL (8.4 - 10.5), BNP 97 pg/mL $(<100)$, troponin I $0.03 \mathrm{ng} / \mathrm{mL}(<0.034), \mathrm{B} 12$ at $401 \mathrm{pg} / \mathrm{mL}$ (180 - 914), prealbumin $16(17-38)$, lactic acid $3.3 \mathrm{mmol} / \mathrm{L}$ (0.5 - 2.2), AST 65 U/L (15 - 46), ALT 103 U/L (13 - 69), ANA negative and HIV negative.

EKG showed sinus tachycardia with a rate of $130 \mathrm{bpm}$ and no acute ST/T wave changes. Portable chest X-ray revealed mild cardiomegaly with no other acute findings. Echocardiogram was consistent with moderate to severely reduced left ventricular systolic function with estimated LV ejection fraction at 30-35\%. Severe anteroseptal hypokinesis and reduced left ventricular wall motion were also present.

With the constellation of peripheral neuropathy, ophthalmoplegia, unexplained cardiomyopathy and lactic acidosis, thiamine deficiency was suspected. After thiamine levels were obtained for testing, she was empirically initiated on intravenous thiamine replacement therapy. Her thiamine level pre-therapy was found to be less than $7 \mathrm{nmol} / \mathrm{L}$ (normal 9 - $14 \mathrm{nmol} / \mathrm{L})$, consistent with the diagnosis of beriberi.

After 5 days of intravenous thiamine therapy in conjunction with physical rehab, the patient's lower extremity weakness significantly improved. She regained the ability to walk with assistance. In addition, her palpitations and shortness of breath subsided and her heart rate decreased to a rate less than $100 \mathrm{bpm}$. During her treatment, the patient had a nutrition consult and was educated on vitamin deficiencies. She went on to agree with G-tube placement and later was discharged home with tube feeding and physical therapy. At the 3-month follow-up, the patient had 5/5-muscle strength in all four extremities. A follow-up echocardiogram revealed normal left ventricular function with an ejection fraction at $60-65 \%$ and normal ventricular wall motions.

\section{Discussion}

Thiamine, also known as vitamin B1, is a water-soluble nutrient that is absorbed in the jejunum of the small intestine, where thiamine phosphorylated and converted into thiamine pyrophosphate (TPP). TPP is the biologically active form of thiamine and serves as a cofactor for several enzymes important in the biosynthesis of essential neurotransmitters and carbohydrate catabolism $[1,2]$. Since the body cannot produce thiamine, the supply lies solely on intake. The amount of the nutrient stored is limited due to the short half-life of 9 - 18 days and continuous excretion through the kidneys [3]. Deficiency can be caused either by inadequate absorption through diet or excess use/depletion; the latter may result from a high carbohydrate diet, hyperthyroidism, pregnancy, lactation, or fever. Chronic or prolonged diarrhea may also impair absorption and increase the depletion of thiamine. While deficiency due to lack of dietary intake is rare in the United States, it can be seen in patients on total parental nutrition without thiamine supplementation [4], and those at a constant alcoholic state or with frequent diet interruption secondary to severe illness. The patient described in this case report developed thiamine deficiency due to her severely decreased oral intake.

Thiamine is involved in numerous body functions, including the nervous and musculoskeletal systems. It is critical for the transport of electrolytes in and out of nerve and muscle cells $[1,3]$. TPP, the active form of thiamine, serves as a cofactor for several enzymes involved primarily in carbohydrate catabolism, and biosynthesis of a number of neurotransmitters including acetylcholine important for neuromuscular transmission [5].

Thiamine deficiency, or beriberi, refers to a Sinhalese phrase that is translated to "I cannot, I cannot" in reference to the profound weakness that may accompany the disorder [6]. The major manifestations of thiamine deficiency involve the cardiovascular system (wet beriberi) and nervous system (dry beriberi).

One of the neurological manifestations of dry beriberi is peripheral neuropathy. This is characterized by a symmetrical impairment of sensory, motor and reflex functions of the extremities with more pronounced effect in the distal limbs. In this patient, acute nutritional thiamine deficiency caused a rapid development of muscle weakness which can be confused with Guillain-Barre syndrome [7]. Several cases of dry beriberi were previously reported in alcoholic patients with concurrent liver damage. In these patients, symptoms of thiamine deficiency are exacerbated by the inadequate liver function and poor diet, and quite often they present with impaired sensory symptoms in additional to muscle weakness [8]. Another presentation of neurologic involvement is Wernicke encephalopathy. This syndrome involves an orderly sequence of symptoms, such as vomiting, nystagmus, palsies of eye movement, fever and ataxia. Eventual progression into mental impairment is considered Korsakoff syndrome [9]. The mechanism stems from the role of thiamine in glucose metabolism. Without their sole energy source of glucose, neurons cannot sustain their cellular functions and begin to die causing CNS symptoms. Improvement and recovery cannot be achieved with thiamine supplementation in Korsakoff syndrome. Therefore, it is critical to recognize the problem and treat those patients in early stage to prevent this irreversible neurological deficit. In our patient, she exhibited mild ptosis and nystagmus without any mental dysfunction. Her early replacement of IV thiamine not only reversed her profound muscle weakness and also resulted in complete resolution of her ptosis and nystagmus, avoided further damage to the central nervous system. 
Wet beriberi is the term used for thiamine deficiency with cardiovascular involvement [10]. The clinical presentation of wet beriberi can be quite varied. The chronic type or slowly developing wet beriberi often presents with peripheral vasodilation, leading to a high cardiac output state [11]. This decreased vascular resistance leads to fluid retention mediated through the renin-angiotensin-aldosterone system. The resulting fluid overload worsens the peripheral edema in dependent areas such as the sacrum. As this condition progresses, the heart already exposed to a defect in energy production from the thiamine deficiency [12], has to work increasingly harder to meet end organ requirements. These overuse injuries eventually result in the physical symptoms of tachycardia, edema, high arterial and venous pressures. This type of wet beriberi presentation is mostly found in the elderly, the chronic ill [6] or alcohol-dependent patients [13] who develop thiamine deficiency symptoms over a period of time. Because of its gradual onset with multiple symptoms and complicated medical histories, the clinician can often miss the diagnosis. Another more rapid type of wet beriberi is classified as acute fulminant cardiovascular beriberi, or Shoshin beriberi [14]. In this form, the predominant injury is to the heart. It is characterized by an acute onset of hypotension, tachycardia and lactic acidosis [15]. In contrast to the chronic type of wet beriberi, edema may not be seen; however, there is left ventricular dysfunction presenting with low ejection fraction. If left untreated, patients die quickly from circulatory collapse and pulmonary edema [16]. The patient presented in this case exhibited similar symptoms as a rapid type of wet beriberi, such as tachycardia, short of breath, systolic murmur, rales and elevated lactic acid in the absent of leg edema. More importantly, her echocardiogram showed significant cardiomyopathy with decreased ejection fraction. After a few days of intravenous Thiamine treatment and supportive care, her shortness of breath and tachycardia resolved. A follow-up echocardiogram showed normal motility and normal ejection fraction.

Because thiamine deficiency can cause cardiomyopathy, the role of thiamine supplement in the management of existing heart failures has been studied. CHF is a major public concern in the United States. Research has shown that patients with heart failure are at higher risk of developing multiple-nutrient deficiencies including B vitamins [17]. In elderly patients with heart failure, loop diuretics to manage fluid and sodium levels can cause hyper-excretion of thiamine [18], thereby increasing the risk of developing thiamine deficiency. Clinical data have shown that thiamine correction in depleted patients can improve left ventricular function as well [19-21]. Therefore, prevention of thiamine deficiency should be a routine component in the overall management of this disease [21]. Furthermore, screening for thiamine deficiency in patients with heart failure should be a regular part of their physician wellness visits especially in those that are elderly, chronically ill, have CHF or on loop diuretics [1].

\section{Conclusion}

In the United States today, vitamins deficiencies such as beriberi are often considered uncommon. This may lead to the under recognition of a severe disease that is very treatable. Neurological disturbances and cardiac failure secondary to thiamine deficiency can be rapidly reversed if caught early and treated properly. The clinicians should have a greater index of suspicion in cases of rapid progressing in polyneuropathy and cardiomyopathy, particularly in a population of alcoholism and malnutrition. Confirmation of low thiamine levels by requesting the assay prior to thiamine replacement ensures an accurate diagnosis and appropriate ongoing treatment. Thiamine deficiency can also occur in patients with heart failure because of confluence factors including poor nutrition and increased urinary losses in tandem with diuretics. With these factors in mind, physicians should suspect thiamine deficiency more often and when confirmed, treat the patients accordingly.

\section{References}

1. Wooley JA. Characteristics of thiamin and its relevance to the management of heart failure. Nutr Clin Pract. 2008;23(5):487-493.

2. Thiamine. Monograph. Altern Med Rev. 2003;8(1):5962.

3. McCormick D, Chong H, Hobbs C, Datta C, Hall PA. Detection of the Ki-67 antigen in fixed and wax-embedded sections with the monoclonal antibody MIB1. Histopathology. 1993;22(4):355-360.

4. Francini-Pesenti F, Brocadello F, Manara R, Santelli L, Laroni A, Caregaro L. Wernicke's syndrome during parenteral feeding: not an unusual complication. Nutrition. 2009;25(2):142-146.

5. Singleton CK, Martin PR. Molecular mechanisms of thiamine utilization. Curr Mol Med. 2001;1(2):197-207.

6. Alvarez CA, Shaw BI, Brown TM. Forgotten but not gone? A probable case of wet beriberi. Federal Practitioner. 2011;18-23.

7. Murphy C, Bangash IH, Varma A. Dry beriberi mimicking the Guillain-Barre syndrome. Pract Neurol. 2009;9(4):221-224.

8. Howard AJ, Kulkarni O, Lekwuwa G, Emsley HC. Rapidly progressive polyneuropathy due to dry beriberi in a man: a case report. J Med Case Rep. 2010;4:409.

9. Zuccoli G, Pipitone N. Neuroimaging findings in acute Wernicke's encephalopathy: review of the literature. AJR Am J Roentgenol. 2009;192(2):501-508.

10. Gubbay ER. Beri-Beri heart disease. Can Med Assoc J. 1966;95(1):21-27.

11. Mehta PA, Dubrey SW. High output heart failure. QJM. 2009;102(4):235-241. 
12. Aldinger EE. Effect of Thiamine Deficiency on Potential Myocardial Contractility. Circ Res. 1965;16:238243.

13. Van der Werff ASA, Klooster A. Relationship of alcohol intake and thiamine deficiency in heart failure. OA Alcohol. 2013;1(1):4-5.

14. Phua KH, Goh LG, Koh K, Ong CN, Tan TC, Wong ML, Lee HP. Thiamine deficiency and sudden deaths: lessons from the past. Lancet. 1990;335(8703):14711472.

15. Ozawa H, Homma Y, Arisawa H, Fukuuchi F, Handa S. Severe metabolic acidosis and heart failure due to thiamine deficiency. Nutrition. 2001;17(4):351-352.

16. Essa E, Velez MR, Smith S, Giri S, Raman SV, Gumina RJ. Cardiovascular magnetic resonance in wet beriberi. J Cardiovasc Magn Reson. 2011;13:41.

17. Azizi-Namini P, Ahmed M, Yan AT, Keith M. The role of $\mathrm{B}$ vitamins in the management of heart failure. Nutr
Clin Pract. 2012;27(3):363-374.

18. Yui Y, Itokawa Y, Kawai C. Furosemide-induced thiamine deficiency. Cardiovasc Res. 1980;14(9):537-540.

19. Shimon I, Almog S, Vered Z, Seligmann H, Shefi M, Peleg E, Rosenthal T, et al. Improved left ventricular function after thiamine supplementation in patients with congestive heart failure receiving long-term furosemide therapy. Am J Med. 1995;98(5):485-490.

20. Mendoza CE, Rodriguez F, Rosenberg DG. Reversal of refractory congestive heart failure after thiamine supplementation: report of a case and review of literature. J Cardiovasc Pharmacol Ther. 2003;8(4):313-316.

21. Schoenenberger AW, Schoenenberger-Berzins R, der Maur CA, Suter PM, Vergopoulos A, Erne P. Thiamine supplementation in symptomatic chronic heart failure: a randomized, double-blind, placebo-controlled, crossover pilot study. Clin Res Cardiol. 2012;101(3):159164. 